\title{
Molecular Structure Studies of Neuraminidase Inhibitors from Eleusine Aegyptiaca L
}

\author{
K. Poonkuzhali ${ }^{1}$, V.Princy ${ }^{2}$, T.Jebastin ${ }^{2}$, N. Sundara Baalajii ${ }^{2}$ T. Palvannan, ${ }^{1 *}$ \\ ${ }^{1}$ Bioprocess and Engineering Laboratory, Department of Biochemistry, Periyar University, Salem - 636011, \\ Tamil Nadu, India \\ ${ }^{2}$ Laboratory of Structural Biology, Department of Bioinformatics, Bharathiar University, Coimbatore - 641046, \\ Tamil Nadu, India
}

*Corresponding Author: Dr. T. Palvannan, Assistant Professor, Bioprocess and Engineering Laboratory, Department of Biochemistry, Periyar University, Tamil Nadu, India

\begin{abstract}
The aqueous extract of Eleusine aegyptiaca L. displays neuraminidase inhibitory value of 1.014 $\mu \mathrm{m}$. Neuraminidase inhibitors were isolated from aqueous extract of Eleusine aegyptiaca L. viz (-2-(2,4dihydroxy phenyl)-5-hydroxy-8H-pyrano[3,2-g]chromen-4-one (1), 5-Hydroxy-2-(3-hydroxy-phenyl)-6isopropenyl-chromen-4-one (2) and aesculetin (3). An ionization technique in mass spectrometry called Direct Analysis in Real Time Mass Spectrometry combined with a Direct Binding Assay was used to characterize the neuraminidase inhibitors. Minimum free-energy modeling analysis revealed the compounds (1,2 and 3) forms an axis with inter-phenolic ring distances of $1.7 \AA, 1.3 \AA$ and $1.1 \AA$ respectively. Solvent Accessible Surface Area of compound 1 shows the $3 D$ binding region of neuraminidase. Molecular docking was performed to analyze the binding between neuraminidase and compound 1 .
\end{abstract}

Keywords: Neuraminidase; Docking; Inhibitor; DART TOF-MS.

\section{INTRODUCTION}

Neuraminidase (NA) is an enzyme involved in viral replication, spread, pathogenesis and considered to be one of the most promising targets for design of antiviral agents [1]. The pressing need for developing novel antiviral agents for treatment of influenza pandemics may be due to drug-resistant influenza virus strains [2]. Among the NA inhibitors (NAIs), only three of these drugs are available on the market (oseltamivir, zanamivir and peramivir). Nature offer plenty of biosynthesized compounds containing chemical scaffolds of high diversity, which present as an infinite pool of chemical entities for target-oriented drug discovery in the battle against this contagious pathogen [2]. The plants are still important for the discovery of new drugs as provider of the drugs based on secondary compounds from plants [3]. These plants stand for a source of natural antioxidants that might serve as leads for developing novel drugs [4].

To discover new bioactive compounds from plant sources, the extract has submitted to chemical screening hyphenated technique such as LC-MS, GC-MS, NMR and ESI-IM-TOFMS which could become new drugs [5]. The new ionization source in mass spectrometry, termed DART (Direct Analysis in Real Time) [6,7], is combined to a time-of-flight mass spectrometer, making it possible to rapidly and accurately identify the chemical components in botanicals and extracts at atmospheric pressure, typically with no sample preparation or processing requirements. Flavonoids in elderberry fruits that contribute to the reported anti-influenza activities were analyzed through DART TOF-MS analysis [8].

This work addresses the neuraminidase inhibition using Eleusine aegyptiaca L., isolation of compounds by spectrometric methods, identification of inhibition mechanism using DART TOF-MS, Solvent Accessible Surface Area (ASA) analysis and molecular docking of neuraminidase inhibitor.

\section{MATERIALS AND METHODS}

\subsection{General Apparatus and Chemicals}

All the reagent grade chemicals were purchased from Sigma Aldrich Chemicals (Chennai, Tamil Nadu, India). Column chromatography was carried out using 230-400 mesh Silica Gel. Silica gel 
(230-400 mesh, Sigma), SPHERIGEL ODS-C18 HPLC column and Sephadex LH-20 were used for plant compounds isolation. ${ }^{1} \mathrm{H}$ and ${ }^{13} \mathrm{C}$ NMR were obtained on a Bruker AM $500\left({ }^{1} \mathrm{H}\right.$ NMR at 500 $\mathrm{MHz},{ }^{13} \mathrm{C}$ NMR at $125 \mathrm{MHz}$ ) spectrometer. Liquid chromatography-mass spectrometry (LC-MS) analyzes were performed using an LC-MS-UPLC-TQD (ESI and APCI) instrument.

\subsection{Plant Materials and Sample Preparation}

The plant species (Eleusine aegyptiaca) was collected in Salem, Tamil Nadu, India. Voucher specimens of plant species were deposited in the Department of Biochemistry, Periyar University, Salem, Tamil Nadu, India. The aqueous extract of Eleusine aegyptiaca L. was prepared by soxhlet apparatus at $40{ }^{\circ} \mathrm{C}$ for 2 days and the filtrate was stored in a refrigerator [9].

\subsection{Neuraminidase Inhibition Assay}

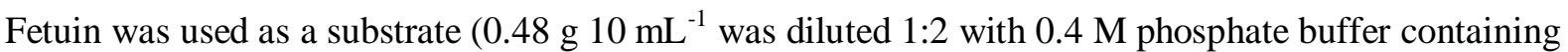
$\mathrm{pH} 5.5,6 \mathrm{mM} \mathrm{Ca}^{2+}$. Aqueous extract of Eleusine aegyptiaca L. $\left(1 \mathrm{mg} \mathrm{mL}^{-1}\right)$ was incubated with neuraminidase $(0.5 \mathrm{U})$ at $37{ }^{\circ} \mathrm{C}$ for $1 \mathrm{~h}$. From these $0.05 \mathrm{~mL}$ of solution was taken for the neuraminidase activity assay. Neuraminidase assay was performed by the method of Aymard Henry, et al. [10]. oseltamivir $\left(1 \mathrm{mg} \mathrm{mL}^{-1}\right)$ was used as a standard inhibitor in the neuraminidase inhibition assay. The mode of inhibition was analyzed using Lineweaver-Burk and Dixon plots [11].

\subsection{Isolation of Neuraminidase Inhibitors from Eleusine Aegyptiaca L. using Comparative Direct Infusion Ion Mobility Mass Spectrometry}

The aqueous extract of Eleusine aegyptiaca L. was subjected to column chromatography on silica gel $(10 \times 30 \mathrm{~cm}, 230-400 \mathrm{mesh}, 150 \mathrm{~g})$ using ethyl acetate $($ EtOAc) and n-butanol $(\mathrm{BuOH})[20: 1(0.5 \mathrm{~L})$, 10:1 (0.5 L), 5:1 $(0.5 \mathrm{~L}), 3: 1(0.5 \mathrm{~L}), 1: 1(0.5 \mathrm{~L})]$ mixtures to give fractions F1-F5. Since F4 possessed promising antineuraminidase activity, it was selected for further studies. Fraction F4 (8 g) was purified by column chromatography using a glass column packed with silica gel and then using ethyl acetate and n-butanol (3:1). Altogether, 10 fractions of $100 \mathrm{~mL}$ each were collected and combined to give six major subfractions (F4.1-F4.6), based on the comparison of TLC profile, subfraction F4.2 (1.3 g) was rechromatographed using the same solvent, to yield compound 1 ( $0.5 \mathrm{~g}$; pale yellow powder) and compound $2(0.8 \mathrm{~g}$; yellowish powder). Subfraction F4.5 (0.9 g) was purified using sephadex LH-20 column chromatography, eluting with water give up compound 3 (0.3 $\mathrm{g}$; yellow needles). All of isolated compounds were identified on the basis of the spectroscopic data. The purity of the compounds was checked by HPLC. HPLC analysis was performed using an Agilent 1100 HPLC-DAD system with a SPHERIGEL ODS-C18 column $(250 \mathrm{~mm} \times 4.6 \mathrm{~mm}$ i.d., $5 \mu \mathrm{m})$ at room temperature. $\mathrm{D}_{2} \mathrm{O}$ water was used as the mobile phase. The flow rate was $0.5 \mathrm{~mL} \mathrm{~min}$, and the eluents were monitored at $260 \mathrm{~nm}$. Mass spectrometry and ion mobility measurements were performed using a Synapt G2 HDMS mass spectrometer (Waters, Manchester, UK). Fraction F4.2 and F4.5 were directly infused for $1 \mathrm{~min}$ via the fluidics system $\left(20 \mu \mathrm{L} \mathrm{min}{ }^{-1}\right)$. Data processing was performed by using HDMS compare software 1.0 and MassLynx 4.1 SCN 779 (Waters) and Sigma Plot software was used for determining the compounds composition [5].

\subsection{DART TOF-MS Analysis of Compounds from Eleusine Aegyptiaca L. and Neuraminidase- Bound Compounds}

The JEOL DART AccuTOF mass spectrometer (JMS-T100LC; Jeol USA, Peabody, MA) was used for chemical analysis of the compounds isolated from aqueous extract of Eleusine aegyptiaca $\mathrm{L}$. and executed in positive ion mode $[\mathrm{M}+\mathrm{H}]+$. The needle voltage was set to $3500 \mathrm{~V}$, heating element to 300 ${ }^{\circ} \mathrm{C}$, electrode $1-150 \mathrm{~V}$, electrode $2-250 \mathrm{~V}$, and helium gas flow to $3.98 \mathrm{~L} / \mathrm{min}$. For the mass spectrometer, the following settings were loaded: orifice 1 set to $20 \mathrm{~V}$, ring lens voltage set to $5 \mathrm{~V}$, and orifice 2 set to $5 \mathrm{~V}$. The peak voltage was set to $1000 \mathrm{~V}$ in order to give peak resolution beginning at $100 \mathrm{~m} / \mathrm{z}$. The microchannel plate detector (MCP) voltage was set to $2550 \mathrm{~V}$. Calibrations were performed internally with each sample using a 10\% (w/v) solution of PEG 600 that provided mass markers throughout the required mass range of 100-1000 amu. Calibration tolerances were held to 5 mmu. Compounds (2-(2,4-dihydroxy-phenyl)-5-hydroxy-8H-pyrano[3,2-g]chromen-4-one (1), 5Hydroxy-2-(3-hydroxy-phenyl)-6-isopropenyl-chromen-4-one (2) and aesculetin (3) as dry powders and incubated with neuraminidase for the Direct Binding Assays) were introduced into the DART He plasma using the closed end of a borosilicate glass melting point capillary tube until a signal was achieved in the total-ion chromatogram (TIC). The next sample was introduced when the TIC 
returned to baseline levels. Chem3D Ultra molecular modeling package was employed for the freeenergy minimizations of the identified compounds using the molecular mechanics two level of theory and accessibility of Solvent Accessible Surface Area [12].

\subsection{Molecular Modelling Analysis}

Molecular docking was performed to the binding mode of Neuraminidase from Clostridium perfringeus with three synthesized compounds from Eleusine aegyptiaca. Schrodinger is commercial docking software used to dock.

\section{RESULTS AND DISCUSSION}

\subsection{Effect of aqueous extract of Eleusine aegyptiaca L. on the activity of neuraminidase}

In the screening for identifying neuraminidase inhibitor, we noted that aqueous extract of Eleusine aegyptiaca $\mathrm{L}$. showed significant inhibition of the hydrolysis of neuraminic acid. As shown in Table 1, the aqueous extract of Eleusine aegyptiaca $\mathrm{L}$. displays inhibitory value $\left(\mathrm{IC}_{50}\right)$ of $1.014 \mu \mathrm{m}$ on neuraminidase. Further, when concentrating the aqueous extract of Eleusine aegyptiaca L., the neuraminidase activity rapidly decreased. The neuraminidase inhibition mechanism (type of inhibition and $\mathrm{K}_{\mathrm{i}}$ value) explained by aqueous extract of Eleusine aegyptiaca $\mathrm{L}$. The aqueous extract of Eleusine aegyptiaca $\mathrm{L}$. showed competitive inhibition of neuraminidase.

\subsection{Isolation of neuraminidase inhibitors from aqueous extract of Eleusine aegyptiaca $L$.}

The aqueous extract of E. aegyptiaca L. has been selected for purification through activity (against neuraminidase)-guided fractionation. Repeated column chromatography of this extract provided two flavonoids $(1 \& 2)$ and one coumarin (3). The purity of the compounds isolated were compound 1 (95.2\%), compound $2(98.5 \%)$ and compound 3 (94.7\%), respectively, as determined by HPLC (Fig. S1). The spectroscopic data of compounds (1-3) has shown in Fig. S2-10.

Chromatogram of aqueous extract of E. aegyptiaca L. got from LC-MS has shown in Fig. S4,7,10 and it revealed neuraminidase inhibitors viz 2-(2,4-dihydroxy-phenyl)-5-hydroxy-8H-pyrano[3,2$g$ ]chromen-4-one (1), 5-Hydroxy-2-(3-hydroxy-phenyl)-6-isopropenyl-chromen-4-one (2) and aesculetin (3). These compounds were responsible for the antineuraminidase activity of aqueous extract of E. aegyptiaca L. Compound 1 and 2 have a basic structure of flavanoid and it is reactive toward neuramindase inhibition. Compound 3 has identified as aesculetin, a derivative of coumarin [6]. The LC separation ensured acceptable resolution for almost all compounds; besides its antineuraminidase property several flavonoids and alkaloids have anti-influenza effects. The structural characteristics of flavonoids from the root of Cudrania tricuspidata and Sophora flavescens required for neuraminidase inhibition has studied and compared with commercially available flavonoids [11,12]. Four coumarins has isolated from methanol extracts of Glycyrrhiza uralensis roots and they have neuraminidase inhibitory activity [13].

The compounds 1-3, were hardly detectable using ESI-IM-TOFMS from infusion of the subfraction F4.2 and F4.5 (aqueous extract of Eleusine aegyptiaca L.) in the mass range from m/z 100-400. The drift time for all relevant signals in this range was between 2.15 and $4.68 \mathrm{~ms}$ for the compounds 1-3. The differences between both fractions has presented in Fig. 1, which compares by a difference plot. In this plot, the mass range $\mathrm{m} / \mathrm{z} 270-350$ has stronger expressed in the subfraction F4.2, whereas the mass range $\mathrm{m} / \mathrm{z}$ 150-190 has stronger expressed in the subfraction F4.5. Compounds 1 and 2 were responsible for the differences in the mass range of $\mathrm{m} / \mathrm{z} 270-350$ (calculated for $\left[\mathrm{C}_{18} \mathrm{H}_{12} \mathrm{O}_{6}\right]^{+} 324.28$ and $\left[\mathrm{C}_{18} \mathrm{H}_{14} \mathrm{O}_{4}\right]^{+}$294.30). The most abundant signal $\mathrm{m} / \mathrm{z} 178.14$ has identified as the compound 3 $\left[\mathrm{C}_{9} \mathrm{H}_{6} \mathrm{O}_{4}\right]^{+}$.

For a direct relative quantitative comparison of both fractions, IM-TOFMS data from the HDMS compare software has exported into Sigma Plot for further data processing provides drift time, accurate mass and areas of every $\mathrm{m} / \mathrm{z}$. The corresponding areas for compounds 1-3 has summed up. The compound $1(49 \%)$ and $2(28 \%)$ concentration was high, and relative low amount of compound 3 $(23 \%)$ has detected in the infusion of both fractions. 


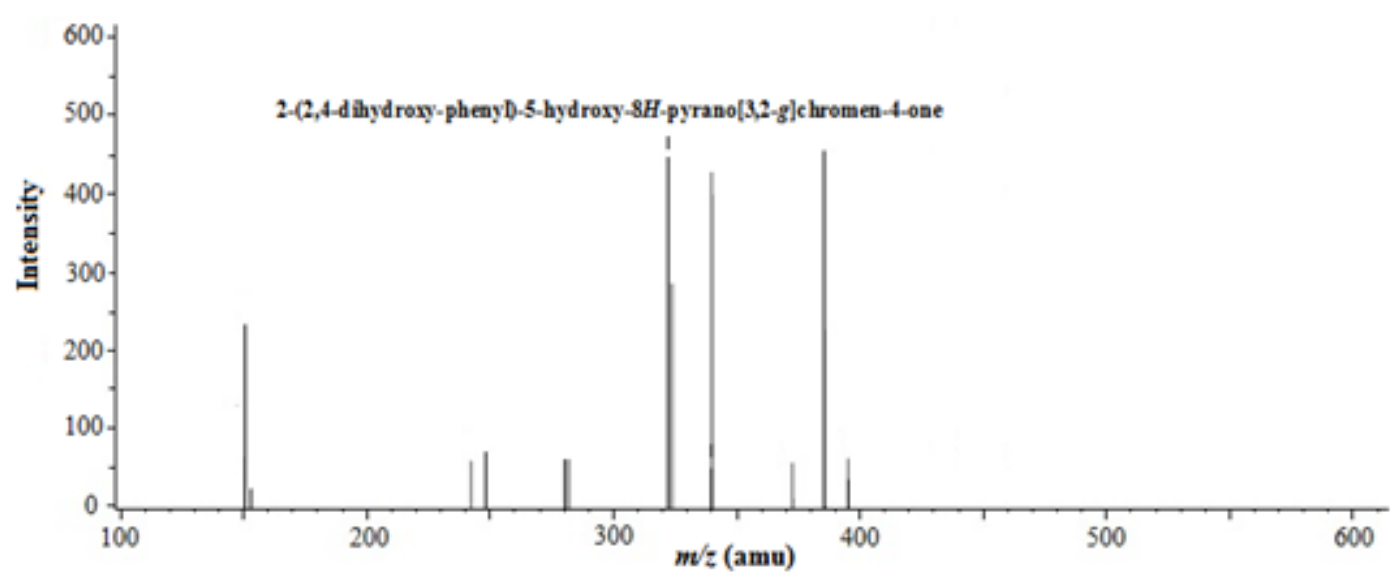

a)

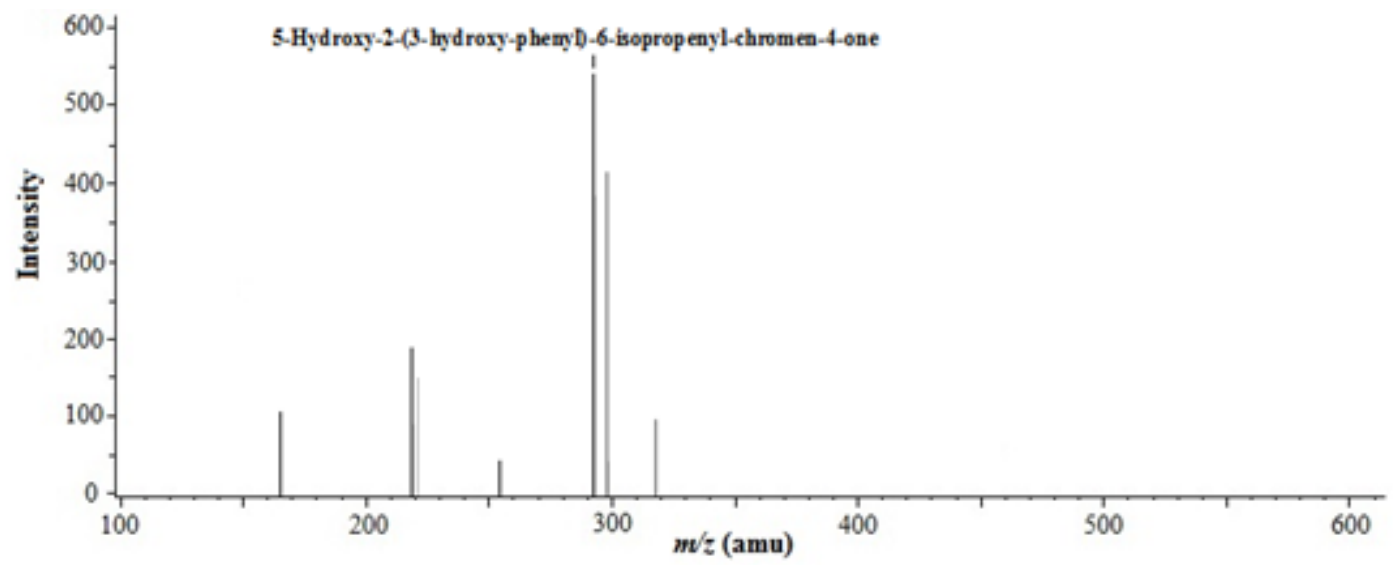

b)

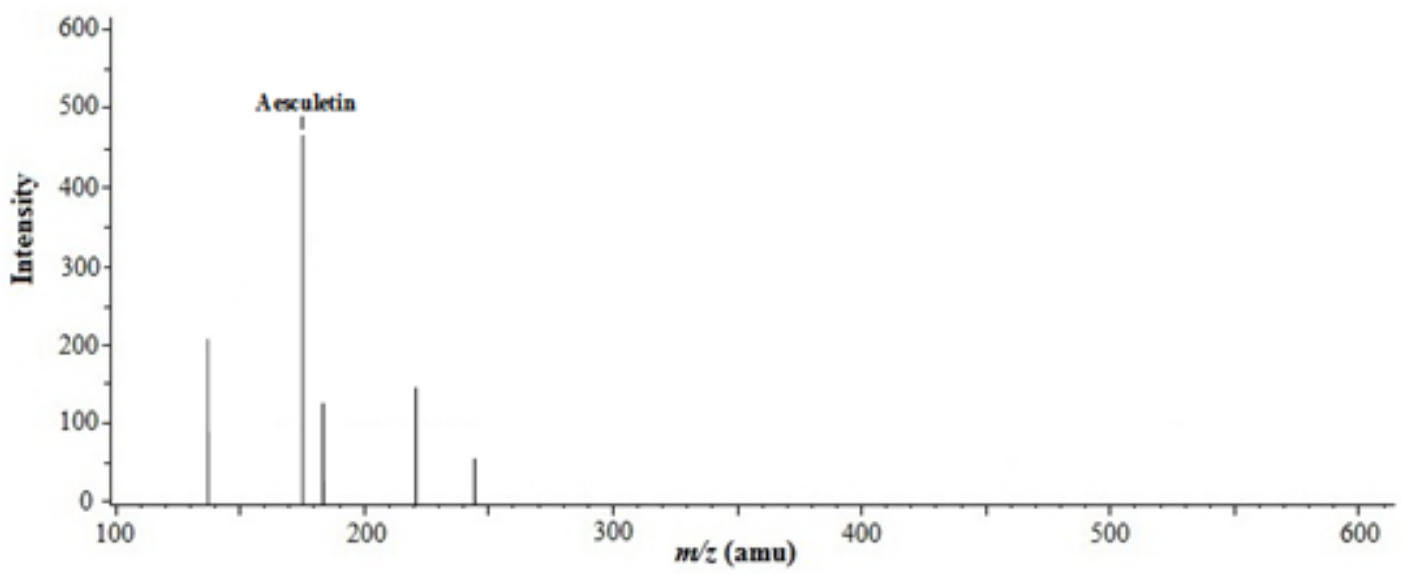

c)

Figure1. DART TOF-MS fingerprints of a) 2-(2,4-dihydroxy-phenyl)-5-hydroxy-8H-pyrano[3,2-g]chromen-4one (1) $(\mathrm{m} / \mathrm{z}=324.1422)$, b) 5-Hydroxy-2-(3-hydroxy-phenyl)-6-isopropenyl-chromen-4-one $(2)(\mathrm{m} / z=$ 293.7359) and c) Aesculetin (3) $(\mathrm{m} / \mathrm{z}=178.2036)$ bound to neuraminidase.

\subsection{Identification of Inhibitors from Eleusine Aegyptiaca L. that Binds to Neuraminidase Using DART TOF-MS}

Fig. 1 shows the DART TOF-MS fingerprint of the compounds those bounds to neuraminidase. This has been accomplished by incubating the compounds with neuraminidase and removing the unbound components by washing through a membrane filter. The unbound neuraminidase were present in the washing medium, did not posses any antineuraminidase activity. This proves that compounds interacting neuraminidase having neuraminidase inhibition property. The structures of compounds 1 , 2 and 3 has confirmed by: (1) measuring the accurate isotopic abundance ratios; (2) determining the 
precise molecular formula based on these isotopic ratios; (3) monitoring the DART-generated fragmentation of these compounds bound to neuraminidase; and (4) molecular modeling of various proposed structures based on the determined molecular formulae [12,14].

The DART ion source and the AccuTOF mass spectrometer were operated in positive-ion mode (metastable helium ionizes atmospheric water, which then donates a proton to the sample). The first flavonoid (1) at $\mathrm{m} / \mathrm{z}[\mathrm{M}+\mathrm{H}]+=324.14 \mathrm{amu}$ (with corresponding DART-generated fragments at $\mathrm{m} / \mathrm{z}$ $[\mathrm{M}+\mathrm{H}]+=325.02,340.56,373.72$, and $281.31 \mathrm{amu}$, representing the formation of methyl ester group in 3-phenyl chromen-4-one has identified as 2-(2,4-dihydroxy-phenyl)-5-hydroxy-8H-pyrano[3,2g]chromen-4-one (1) (Fig. 1a). The second flavonoid identified 5-Hydroxy-2-(3-hydroxy-phenyl)-6isopropenyl-chromen-4-one (2) at $\mathrm{m} / \mathrm{z}[\mathrm{M}+\mathrm{H}]+=293.73 \mathrm{amu}$ has methylated due to formation of free isopropenyl ring at $6^{\text {th }}$ position of basic flavanoid ring structure (Fig. 1b). Fig. 1c shows that the aesculetin (3) at $\mathrm{m} / \mathrm{z}[\mathrm{M}+\mathrm{H}]+=178.20 \mathrm{amu}$ describes the loss of water from the parent coumarin structure at hydroxyl groups on $6 \& 7^{\text {th }}$ position. The compounds isolated has subjected to focus on inhibition assay against the neuraminidase, and compound 1 achieved high inhibition power at a concentration of $1.27 \mu \mathrm{g} / \mathrm{ml}$ (1) compared with oseltamivir.

\subsection{Molecular Structure Studies}

Minimum free-energy modeling analysis revealed the compounds 1, 2 and 3 forms an axis with interphenolic ring distances of $1.7 \AA, 1.3 \AA$ and $1.1 \AA$, respectively (Fig. 2). This distance is well within the size constraints of the neuraminidase (NA) binding domain pocket ( $3.5 \AA$ ) of influenza viruses, which is responsible for host cell receptor binding and viral entry [15]. The phenolic regions of compounds, most likely bind to the neuraminidase binding domain because of its size, potentially decreasing the receptor cleavage of neuraminidase and it facilitates the movement of virus [16].

The solvent accessible surface area (ASA) describes the area over which contact between protein and solvent can occur. Chem3D Ultra offers a powerful method for selecting which atoms has included in the surface of protein. The user is able to specify which atoms of protein are to have their area calculated, which are to be included (show as pink color), and which are to be excluded entirely (other than pink color and thus play no part at all in the calculations). The output of ASA of compound 1 has analyzed and matched by radius $(1.7 \AA)$ taken from the literature, proves that main chain alpha carbon and all side chain atoms and groups is responsible for the binding with neuraminidase.

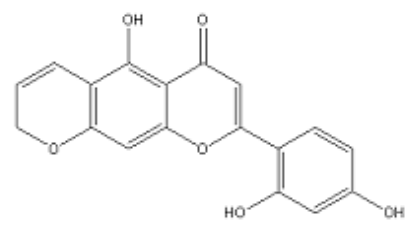

(1)
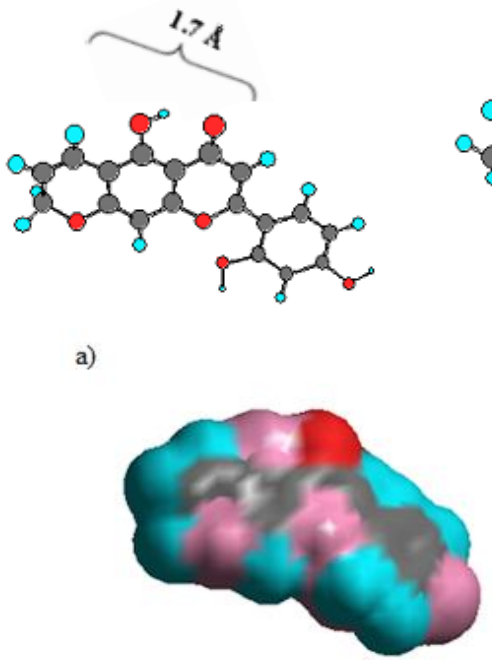

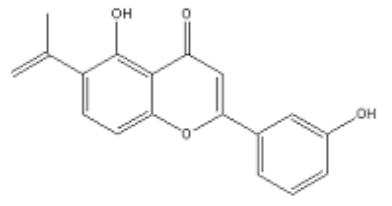

(2)

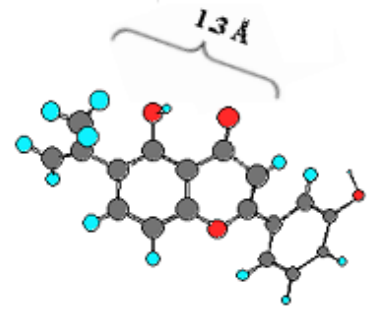

b)

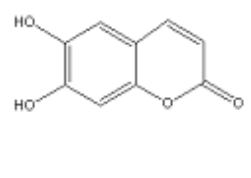

(3)
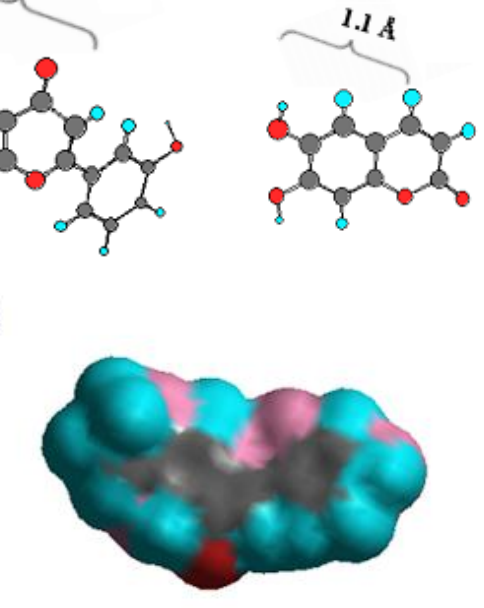

Figure2. The structures of compounds (1), (2) and (3). The region most likely to bind to the neuraminidase bracketed in the free-energy minimized three-dimensional structure of 1, 2 and 3. Compound $13 D$ structure of solvent accessible surface for neuraminidase binding a) front side b) back side. 
a)

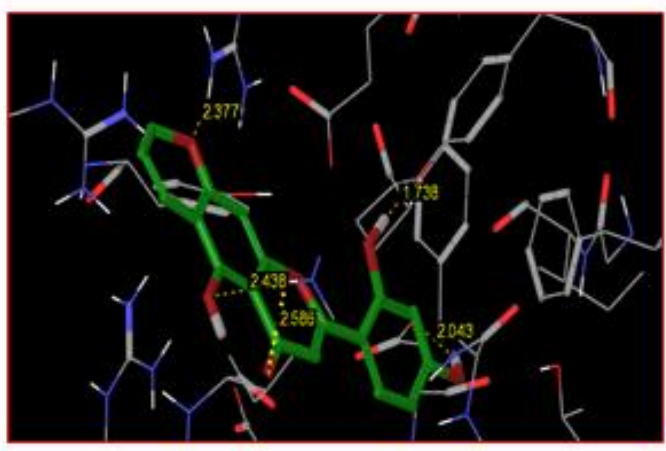

c)

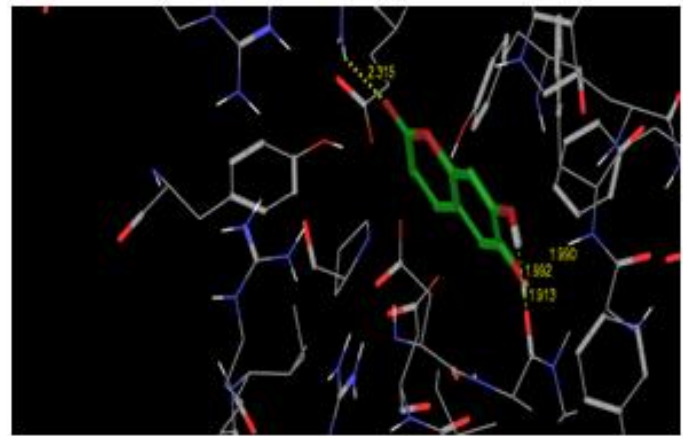

b)

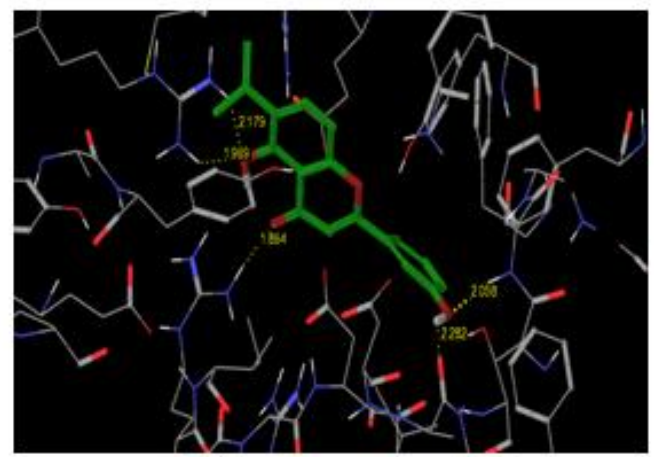

d)

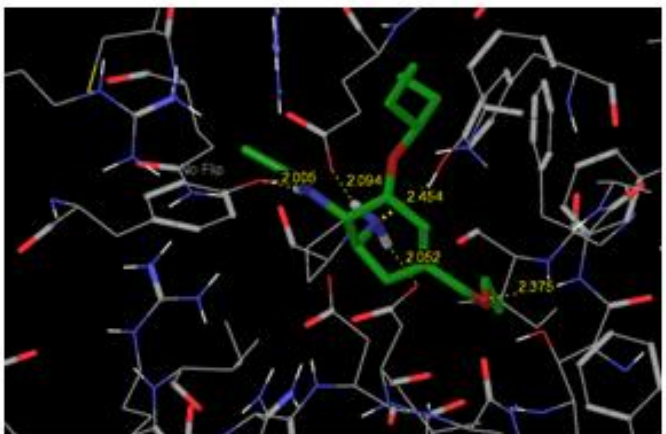

Figure3. Hydrogen bond interactions between compound 1 (a), 2 (b) 3 (c), Oseltamivir and neuraminidase (d)

\subsection{Docking Study}

Docking has performed neuraminidase from Clostridium perfringeus with compound 1 . The interaction of the compound 1 with neuraminidase has shown in fig. 3 and the number of hydrogen bond, docking score and residues of interaction has also noted. Compound 1 has the excellent docked binding energy of $-6.20 \mathrm{Kcal} / \mathrm{mol}$ and forms five hydrogen bonds with active site residues TRP1354, TRP1354, ARG1555, TYR1485 and ASP1328 respectively (Table 1). Through the docking, we have showed the new inhibitor shows much better result for inhibition of neuraminidase.

Table1. Docking of isolated compounds and Oseltamivir with neuraminidase results in G-score, hydrogen bond, distance and interaction residues

\begin{tabular}{|c|c|c|c|}
\hline G-score $(\mathrm{Kcal} / \mathrm{mol})$ & Number of Hydrogen bonds & Distance $(\AA)$ & Interaction \\
\hline \multirow{5}{*}{$\begin{array}{l}\text { Compound } 1 \\
-6.20\end{array}$} & \multirow[t]{5}{*}{5} & 2.438 & TRP $1354(\mathrm{O} \ldots . . \mathrm{H})$ \\
\hline & & 2.586 & TRP $1354(\mathrm{O} \ldots . . \mathrm{H})$ \\
\hline & & 2.377 & ARG1555 (H....O) \\
\hline & & 1.738 & TYR $1485(\mathrm{O} \ldots \mathrm{H})$ \\
\hline & & 2.043 & ASP $1328(\mathrm{O} \ldots . . \mathrm{H})$ \\
\hline \multirow[t]{5}{*}{ Compound $2-5.96$} & \multirow[t]{5}{*}{5} & 1.864 & ARG $1266(\mathrm{O} \ldots \mathrm{H})$ \\
\hline & & 2.179 & ARG $1615(\mathrm{O} \ldots \mathrm{H})$ \\
\hline & & 1.969 & ARG $1615(\mathrm{O} \ldots . . \mathrm{H})$ \\
\hline & & 2.058 & PHE $1353(\mathrm{O} \ldots \mathrm{H})$ \\
\hline & & 2.282 & ALA $1292(\mathrm{O} \ldots \mathrm{H})$ \\
\hline \multirow[t]{4}{*}{ Compound $3-4.20$} & \multirow[t]{4}{*}{4} & 1.913 & ALA $1292(\mathrm{H} \ldots \mathrm{O})$ \\
\hline & & 1.992 & ALA $1292(\mathrm{H} . . . \mathrm{O})$ \\
\hline & & 1.990 & PHE $1353(\mathrm{H} . . . . \mathrm{O})$ \\
\hline & & 2.315 & ARG $1555(\mathrm{H} \ldots . . \mathrm{O})$ \\
\hline \multirow[t]{5}{*}{ Oseltamivir -4.39} & \multirow[t]{5}{*}{5} & 2.375 & PHE $1353(\mathrm{O} \ldots . . \mathrm{H})$ \\
\hline & & 2.005 & TYR1655 (O....H) \\
\hline & & 2.094 & GLU1539 (O...H) \\
\hline & & 2.052 & ASP $1328(\mathrm{O} \ldots . . \mathrm{H})$ \\
\hline & & 2.454 & TYR $1485(\mathrm{O} \ldots . . \mathrm{H})$ \\
\hline
\end{tabular}




\section{CONCLUSION}

Neuraminidase shows competitive inhibition for this plant species. The identified and isolated compounds were (-2-(2,4-dihydroxy phenyl)-5-hydroxy-8H-pyrano[3,2-g]chromen-4-one (1), 5Hydroxy-2-(3-hydroxy-phenyl)-6-isopropenyl-chromen-4-one (2) and aesculetin (3). The molecular mode-of-action of these flavonoids was determined by direct binding to neuraminidase resulting in the inability of viruses to enter host cells. From our study results, Eleusine aegyptiaca L. shows contribution with ethnopharmacological knowledge about antiviral effects of precious hints for the selection of promising natural materials.

\section{ACKNOWLEDGEMENTS}

The first author (INSPIRE Fellow Code, IF10343/No.DST/INSPIRE Fellowship/2010) is grateful to Government of India, Ministry of Science \& Technology, Department of Science \& Technology, New Delhi, India for financial support. The authors were thankful to IIT Chennai and CDRI Lucknow for the analysis support.

\section{REFERENCES}

[1] J.A. Vilensky, P. Foley, S. Gilman, Children and encephalitis lethargica: a historical review, Pediatr. Neurol. 37 (2007) 79-84.

[2] G. Ulrike, S. Michaela, G. Susanne von, K. Johannes, R.L. Klaus, M.R. Judith, Influenza neuraminidase: a druggable target for natural products, Nat. Prod. Rep. 29 (2012) 11-36.

[3] K.O. Ganiyat, A.O. Patricia, S. Julius, O. Olu waseyi, T. Emmanuel, Emmanuel T, Phytochemical screening, antimicrobial and antioxidant activities of four Nigerian medicinal plants, Ann. Biol. Res. 1 (2010) 114-120.

[4] F. Conforti, S. Sosa, M. Marrelli, F. Menichini, G.A. Statti, D. Uzunov, In vivo anti-inflammatory and in vitro antioxidant activities of Mediterranean dietary plants, J. Ethnopharm. 116 (2008) 144-151.

[5] T. Stark, S. Marxen, A. Rutschle, G. Lucking, S. Scherer, M. Ehling-Schulz, T. Hofmann, Mass Spectrometric Profiling of Bacillus Cereus Strains and Quantitation of the Emetic Toxin Cereulide by Means of Stable Isotope Dilution Analysis and HEp-2 Bioassay, Anal. Bioanal. Chem. 405 (2013) 191201.

[6] J. Wolfender, N. Karine, K. Hostettmann, Liquid chromatography with ultraviolet absorbance-mass spectrometric detection and with nuclear magnetic resonance spectroscopy: a powerful combination for the on-line structural investigation of plant metabolites, J. Chromatogr. A. 1000 (2003) 437-455.

[7] R.B. Cody, J.A. Laramee, H.D. Durst, Versatile new ion source for the analysis of materials in open air under ambient conditions, Anal. Chem. 77 (2005) 2297-2302.

[8] J.R. Bill Roschek, C. Ryan Fink, Matthew D. McMichael, L. Dan, S. Randall Alberte, Elderberry flavonoids bind to and prevent H1N1 infection in vitro, Phytochemistry 70 (2009) 1255-1261.

[9] K.M. Hazra, R.N. Roy, S.K. Sen, S. Laskar, Isolation of antibacterial pentahydroxy flavones from the seeds of Mimusops elengi Linn, Afr. J. Biotechnol. 6 (2007) 1446-1449.

[10] M. Aymard Henry, M.T. Coleman, W.R. Dowdle, W.G. Laver, G.C. Schild, R.G. Webster, Influenza neuraminidase and neuraminidase inhibition test procedure, Bull. Org. Mond Sante \& Bull. Wld. Hlth. Org. 48 (1973) 199-202.

[11] R. Young Bae, J. Marcus Curtis-Long, J. Won Lee, J. Hyo Kim, J. Young Kim, K. Young Kang, W. Song Lee, K. Hun Park, Characteristic of neuraminidase inhibitory xanthones from Cudrania tricuspidata, Bioorg. Med. Chem. 17 (2009) 2744-2750.

[12] R. Young Bae, J. Marcus Curtis-Long, J. Hyo Kim, S. Hun Jeong, M. Suk Yang, K. Woo Lee, W. Song Lee, K. Hun Park, Pterocarpans and flavanones from Sophora flavescens displaying potent neuraminidase inhibition, Bioorg. Med. Chem. Lett. 18 (2008) 6046-6049.

[13] Y.B. Ryu, J.H. Kim, S.J. Park, J.S. Chang, M.C. Rho, K.H. Bae, K.H. Park, W.S. Lee, Inhibition of neuraminidase activity by polyphenol compounds isolated from the roots of Glycyrrhiza uralensis, Bioorg. Med. Chem. Lett. 20 (2010) 971-974. 
[14] K.P. Madhusudanan, S. Banerjee, S.P. Khanuja, S.K. Chattopadhyay, Analysis of hairy root culture of Rauvolfia serpentina using direct analysis in real time mass spectrometric technique, Biomed. Chromatogr. 22 (2008) 596-600.

[15] Y.M. Knox, K. Hayashi, T. Suzutani, M. Ogasawara, I. Yoshida, R. Shiina, A. Tsukui, N. Terahara, M. Azuma, Activity of anthocyanins from fruit extract of Ribus nigrum L. against influenza A and B viruses, Acta. Virol. 24 (2001) 209-215.

[16] V. Larisa Gubareva, K. Laurent, G. Frederick Hayden, New Drug Classes, Lancet. 355 (2000) 827-835.

Citation: K. Poonkuzhali et al., " Molecular Structure Studies of Neuraminidase Inhibitors from Eleusine Aegyptiaca L", International Journal of Medicinal Plants and Natural Products (IJMPNP), vol. 3, no. 2, pp. 19-26, 2017. http://dx.doi.org/10.20431/2454-7999.0302003

Copyright: (c) 2017 Authors. This is an open-access article distributed under the terms of the Creative Commons Attribution License, which permits unrestricted use, distribution, and reproduction in any medium, provided the original author and source are credited. 